\title{
Unexpected Early Oncologic Mortality after Open Radical Cystectomy for Bladder Cancer: Who Is to Be Blamed?
}

\author{
Richard E. Hautmann ${ }^{a}$ Christian Bolenz ${ }^{a}$ Björn Volkmer ${ }^{b}$

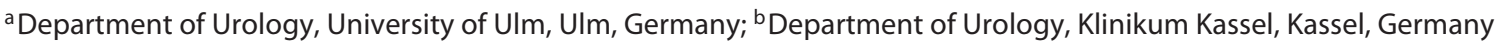

\section{Keywords}

Urinary bladder neoplasms · Cystectomy · Neoplasm

recurrence . Oncologic mortality non urothelial histology, unresectable tumours, atypical occult metastasis), rather than technical factors, had the leading role in EOM. Understaging was universal.

(c) 2019 S. Karger AG, Basel

\section{Introduction}

Perioperative mortality of open radical cystectomy (RC) for bladder cancer (BC) defined as death within 30 days of surgery or prior to hospital discharge has decreased from nearly 20\% before 1970 to approximately $2 \%$ in most contemporary series. When perioperative mortality is defined as death within 60 or 90 days after surgery, mortality rates increase to 2.4 and $3.9 \%[1,2]$. In a most recent report, Groeben et al. [3] have detailed inhospital outcomes after RC for BC in the United States and Germany from 2006 to 2014.

To the best of our knowledge the contribution of early oncologic mortality (EOM), defined as death between $\geq 30$ and $\leq 90$ days after RC to perioperative mortality has never been studied systematically, although every high-volume BC surgeon has encountered similar cases. It is also surprising that almost all Kaplan-Meier plots on cancerspecific survival or disease-specific survival start with an immediate steep decline. Occasionally EOM is followed karger@karger.com

www.karger.com/uin

Karger ${ }^{\prime}=$
(C) 2019 S. Karger AG, Basel

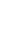

\begin{abstract}
distant failure rate. Conclusions: Disease related (advanced tumour stage, positive soft tissue surgical margins (+STSM),
\end{abstract}

Richard E. Hautmann, MD, hon

Department of Urology, University of Ulm Albert-Einstein-Allee 23

Boschstrasse 4a, DE-89231 Ulm (Germany)

E-Mail Richard.hautmann@uni-ulm.de 
Table 1. Unexpected EOM (>30 till $\leq 90$ days) after open RC for margin positive (R1/R2) or locally advanced or unresectable BC: Group 1

\begin{tabular}{|c|c|c|c|c|c|c|c|c|c|}
\hline Name & $\begin{array}{l}\text { Age, } \\
\text { years }\end{array}$ & Gender & Year & Preoperative stage & Histology & RCX specimen & NAC & Postoperative course & $\begin{array}{l}\text { CSS, } \\
\text { days }\end{array}$ \\
\hline \multicolumn{10}{|c|}{ Local recurrence } \\
\hline S.K. & 78 & Male & 2000 & pT1 cN0 cM0 & UC G2 & $\begin{array}{l}\text { pT4b (rectum) cN0 } \\
\text { cM0 G3 }\end{array}$ & R2 none & $\begin{array}{l}\text { Huge local recurrence, } \\
\text { ileus }\end{array}$ & 65 \\
\hline K.M. & 71 & Female & 1986 & pT2a cN0 cM0 & UC G3 & pT4b pN0 cM0 G3 R2 & None & Huge local recurrence, ileus & 71 \\
\hline T.J. & 46 & Male & 1990 & pT2a cN0 cM0 & UC G3 & pT4b pN2 cM0 G3 R2 & None & $\begin{array}{l}\text { Local recurrence, penile } \\
\text { infilt. invasion pelvic bones }\end{array}$ & 60 \\
\hline J.S. & 44 & Female & 2005 & pT2a cN0 cM0 & UC G4 & pT4b pN3 cM0 G3 R2 & None & $\begin{array}{l}\text { Local recurrence, diffuse } \\
\text { liver mets }\end{array}$ & 46 \\
\hline S.M. & 80 & Male & 1999 & pT4a cN0 cM0 & UC G3 & pT4a pN2 cM0 G3 R2 & None & Local recurrence & 31 \\
\hline H.M. & 18 & Male & 1995 & $\begin{array}{l}\text { Embr. rhabdomyosarcoma, } \\
\text { IV, yM0, pul }\end{array}$ & $\begin{array}{l}\text { Embr. } \\
\text { rhabdomyosarcoma }\end{array}$ & $\begin{array}{l}\text { Stage IV (penetrating } \\
\text { abdominal wall) }\end{array}$ & $\begin{array}{l}\text { Adriamycin } \times 4 \\
\text { ifosfam. DTIC }\end{array}$ & $\begin{array}{l}\text { Local and pulmonary } \\
\text { progression }\end{array}$ & 75 \\
\hline A.H. & 65 & Male & 1987 & pT3a cN0 cM0 & UC G3 & pT4b pN0 cM0 G3 R2 & MVAC $\times 2$ & $\begin{array}{l}\text { Local recurrence, ileus } \\
\text { sepsis }\end{array}$ & 57 \\
\hline K.D. & 55 & Male & 1998 & pT2a cN0 cM0 & UC G3 & pT3b pN1 cM0 G3 R2 & None & Local recurrence, ileus & 82 \\
\hline K.R. & 68 & Female & 2003 & pT4b ycN0 cM0 & UC G2 & pT4b pN2 cM0 G3 R1 & Gem-Cis $\times 4$ & Huge local recurrence & 43 \\
\hline \multicolumn{10}{|c|}{ Pulmonary, hepatic } \\
\hline R.R. & 68 & Male & 1990 & pT2a cN0 cM0 & UC G3 & $\begin{array}{l}\text { pT4b pN2 pM1 (lymph) } \\
\text { G3 R2 }\end{array}$ & None & $\begin{array}{l}\text { Paraneoplastic thrombosis } \\
\text { lethal pulmonaryembolism }\end{array}$ & 63 \\
\hline V.E. & 74 & Female & 1999 & pT2a cN0 cM0 & UC G3 & pT4a cN2 cM1 (pul) G3 R2 & None & Pulmonary mets & 44 \\
\hline M.W. & 69 & Male & 1990 & pT4a cN0 ycM0 & UC G3 & pT4b pN3 cM1 G3 R1 & $\mathrm{EC} \times 6$ & Total liver necrosis sepsis & 30 \\
\hline
\end{tabular}

EOM, early oncologic mortality; BC, bladder cancer; NAC, neoadjuvant chemotherapy; CSS, cancer specific survival.

by queries regarding diagnosis, staging, indication, surgical skill and breach of oncologic principles. We also sought to investigate possible factors contributing to EOM.

\section{Methods}

\section{Patient Population}

Between January 1986 und December 2008, 1,487 patients underwent RC for malignant tumours of the bladder at our institution with curative intent. Patient characteristics were entered into a prospectively maintained institutional review board-approved database. Data was collected from the patient or the treating urologists to document the course of the disease. Neoadjuvant chemotherapy (NAC) was not routinely offered to patients, but we had patients referred for RC who had NAC in the referring institution. Patient characteristics are presented in Tables 1 and 2 and are available in previous publications of our RC/urinary diversion series [4]. Study inclusion criteria were as follows: (1) primary BC treated with open $\mathrm{RC}$ with the intent to cure $(\mathrm{cNO}, \mathrm{cMo})$ and NAC responders (ycN0, ycM0). (2) All perioperative mortalities defined as death within 90 days of surgery were identified. Medical records of these patients were reviewed to determine the cause of death, any precipitating intraoperative or postoperative factors, predisposing comorbidities, and management of early postoperative complications that could have contribute to mortality. (3) Concomitant surgical procedures, intraoperative findings and choice of urinary diversion were also recorded to determine whether disease-related or treatment-related factors were associated with EOM. Exclusion criteria were (1) presence of untreated metastasis (mts) prior to $\mathrm{RC}$ or other primaries or distant mts at diagnosis; (2) perioperative non-oncologic complications leading to death.

\section{Patient-Related Variables}

Clinical staging was initially determined by the treating physician and was verified by the investigators in all 1,487 patients. Staging was performed within 8 weeks before surgery and included ultrasound, intravenous pyelogram, cystoscopy, transurethral tumour resection, bimanual examination under anaesthesia, CT of the chest, CT or MRI of the abdomen and pelvis. Patients were defined as cNO, when all pelvic LN were $<8 \mathrm{~mm}$ and abdominal LNs $<10 \mathrm{~mm}$ in maximum diameter. All surgical specimens were examined under a standardized pathologic protocol. Tumour staging and grading were standardized to the American Joint Committee on Cancer and World Health Organisation recommendations respectively. Initial precystectomy clinical and final post cystectomy pathologic stages were compared to determine tumour upstaging. A positive soft tissue surgical margin (+STSM) was defined as the presence of tumour at inked areas of soft tissue on the RC specimen.

\section{Clinical Outcomes and Analysis}

Patients with +STSM, locally advanced or unresectable pT4 (R1, R2), pN-any, who were never free of disease were assigned to Group 1. Patients with resectable disease (R0) formed Group 2. Cause of death was prospectively entered into the institutional database, verified by chart review and corroborated by death certifi- 
Table 2. Unexpected EOM ( $\geq 30$ till $\leq 90$ days) after open $\mathrm{RC}$ for advanced but resectable BC (R0): Group 2

\begin{tabular}{|c|c|c|c|c|c|c|c|c|c|}
\hline Name & $\begin{array}{l}\text { Age, } \\
\text { years }\end{array}$ & Gender & Year & $\begin{array}{l}\text { Preoperative } \\
\text { stage }\end{array}$ & Histology & RCX specimen & NAC & Postoperative course & $\begin{array}{l}\text { CSS, } \\
\text { days }\end{array}$ \\
\hline \multicolumn{10}{|c|}{ Local recurrence } \\
\hline R.H. & 74 & Male & 1997 & pT2a cN0 cM0 & UC G3 & pT4a cN0 cM0 no PLND & CIS + 45 Gy & Local recurrenc, ileus & 88 \\
\hline \multicolumn{10}{|c|}{ Neurological, metabolic, gastrointestinal } \\
\hline K.H. & 56 & Male & 1988 & pT2a cN0 cM0 & $\begin{array}{l}\text { UC G3 + } \\
\text { neuroendocrine }\end{array}$ & $\begin{array}{l}\text { pT4b pN1 cM0 G3 } \\
\text { neuroendorin }\end{array}$ & MVAC $\times 2$ & Paraplegia, fracture Th12 & 70 \\
\hline B.K. & 69 & Male & 1991 & pT3a cN0 cM0 & UC G3 & pT3a pN1 cM0 G3 & None & $\begin{array}{l}\text { Path.fract.fem., hyperCa. } \\
\text { untreat }\end{array}$ & 60 \\
\hline K.W. & 72 & Male & 1999 & pT1 cN0 cM0 & UC G3 + small cell & pT4b pN3 cM1 (perit, N) G3 & None & Perit. Carcinomatosis, ileus & 58 \\
\hline B.H. & 69 & Male & 1998 & pT2a cN0 cM0 & $\mathrm{UC} \mathrm{G2}$ & pT3b pN2 pM1 (lymph) G3 & None & LN extensiv, progressive & 84 \\
\hline S.H. & 66 & Male & 1995 & pT3b cN0 cM0 & UC G3 & pT3b pN0 cM0 G3 & None & $\begin{array}{l}\text { LN mets, cava compression, } \\
\text { RLND: intestinal necrosis }\end{array}$ & 32 \\
\hline \multicolumn{10}{|c|}{ Brain metastasis } \\
\hline P.H. & 58 & Male & 2000 & pT2a cN0 cM0 & UC G3 & pT3b pN2 cM0 G3 & None & Brain mets, brachiofac. paresis & 31 \\
\hline W.A. & 63 & Female & 2008 & pT1 cN0 cM0 & Carcinosarcoma G3 & pT4a pN0 cM0 G3 & None & Brain mets, stupor & 75 \\
\hline P.H. & 68 & Male & 1996 & pT4a cN0 cM0 & $\begin{array}{l}\text { UC G3 + } \\
\text { neuroendocrine }\end{array}$ & pT4a pN0 cM0 G3 & None & Brain mets, epileptic seizure & 53 \\
\hline D.H. & 42 & Male & 1993 & pT2a cN0 cM0 & UC G3 & pT4a pN2 cM0 G3 & None & Brain mets, epileptic seizure & 30 \\
\hline \multicolumn{10}{|c|}{ Pulmonary/bone metastasis } \\
\hline N.G & 64 & Male & 2007 & pT2a cN0 cM0 & UC G3 & pT3b pN0 cM0 G3 & None & Pulmonary + bone mets & 51 \\
\hline W.W. & 75 & Male & 2003 & pT3b cN0 cM0 & $\begin{array}{l}\text { UC G3 + } \\
\text { neuroendocrine }\end{array}$ & $\begin{array}{l}\text { pT4a pN1 cM0 G3 } \\
\text { neuroendocrine }\end{array}$ & $\begin{array}{l}\text { Rad. } \\
\text { chemo }\end{array}$ & Pulmonary mets & 85 \\
\hline
\end{tabular}

EOM, early oncologic mortality; BC, bladder cancer; NAC, neoadjuvant chemotherapy; CSS, cancer specific survival.

cates. The causes of death were classified into 6 categories - pulmonary, thromboembolic, neurological, gastrointestinal, hepatic and metabolic. Early mortality in cases with neurological, pulmonary, hepatic and metabolic death was easy to classify. It was more difficult to assign the local recurrence (LR) cases of the R2 group. They were classified as EOM when the disease diameter $(\geq 10 \mathrm{~cm})$ and the rapidity of growth were the leading symptom of EOM.

The primary outcome of interest was EOM, defined as any oncology-related mortality within $\geq 30$ and $\leq 90$ days after RC. We choose this time frame as a cut off for EOM, so that mortality was most likely related to oncologic disease severity rather than to the surgical technique or surgical complications.

\section{Results}

i. A total of 1,487 patients, with complete data were included into the study. There was a perioperative mortality of $5.2 \%$ ( $\leq 90$ days). EOM developed in 24 patients $(1.6 \%)$.

ii. Fifteen of the 24 EOM patients with clinically organ confined disease had non-organ confined pathological stage. Eleven patients were pT4b. Twelve patients were never free of disease (Group 1) and 12 were (Group 2) free of disease. Pathological upstaging of the T-category oc- curred in 19 out of 24 patients. When LN involvement was categorized as upstaging, 14 out of 24 patients were upstaged. There was no pathological down staging.

iii. Patients with asymptomatic, very rare or atypical mts escaped the primary clinical staging. An example are the 4 patients with brain mts in Group 2 (Tables 2, 3). Four out of the 24 patients had non-urothelial BC.

iv. Our analysis shows that all LR occurred when there was extra vesical disease at RC. In Group 1, out of 12 patients, 8 had a frozen pelvis at the time of surgery (versus 2/12 in Group 2) and 9 out of 12 patients in Group 1 versus only 1 out of 12 in Group 2 were with an LR. In 7 patients, the LR was the first and only failure without distant mts. Three EOM patients had synchronous local and distant failure.

$\mathrm{v}$. The metastatic pattern of the 2 groups was different. While both had a comparable $\mathrm{pN}+$ rate (7/12 in Group 2 vs. 8/12 in Group 1), Group 1 is characterized by local tumor spread (LR 9/12) and a low distant failure rate (3/12). Group 2 has a low LR rate of 1 out of 12 patients and a distant failure rate of 9 out of 12 patients (Table 3 ).

vi. In none of our EOM patients, breaching of oncologic surgical principles (entry into the bladder or urine 
Table 3. Unexpected EOM ( $\geq 30$ till $\leq 90$ days) and pattern of failure after open RC in locally advanced, margin positive (R1/R2), or unresectable (Group 1) versus resectable (R0), BC (Group 2). (>1 feature may apply per patient)

\begin{tabular}{lll}
\hline & $\begin{array}{l}\text { Group 2 (R0; } \\
n=12 \text { patients) }\end{array}$ & $\begin{array}{l}\text { Group 1 (R2; } \\
n=12 \text { patients) }\end{array}$ \\
\hline pN+ & 7 & 8 \\
pN1 & 3 & 1 \\
pN2 & 3 & 5 \\
pN3 & 1 & 2 \\
pN0 & 5 & 4 \\
pN+ complications & 3 & 2 \\
Frozen pelvis & 2 & 8 \\
Peritoneal carcinomatosis & 1 & - \\
Local recurrence & 1 & 9 \\
Distant failure & 9 & 5 \\
$\quad$ Brain mts & 4 & - \\
Liver mts & - & 2 \\
Pulmonary mts & 2 & 2 \\
Bone mts & 3 & 1 \\
Metabolic & 1 & 1 \\
\hline
\end{tabular}

EOM, early oncologic mortality; RC, radical cystectomy; BC, bladder cancer.

leakage from the urethra or the ureters) could be demonstrated.

vii. Reasons for suboptimal/no NAC were Toxicity (patient A.H.) and patient refusal (P.H., W.W., K.W.)

\section{Discussion}

In our series, 24 out of 93 patients with perioperative mortality had EOM (1.6\%). Previous series of open RC for $\mathrm{BC}$, including our own, did not report EOM systematically [4]. This is most likely because EOM was not evaluated and misclassified into categories like gastrointestinal, sepsis, and so on. The surgical morbidity database of the MSKCC database reports only on 1 out of 1,142 patients who died of disease progression during $\leq 90$ days [5]. In Bern, 35 out of 507 patients died within 3 months after surgery, and reasons for death were tumour related in 12 and non-tumour related in 23 [6]. In contrast to $\mathrm{EOM}$, more information is available on early oncologic failure (EOF) defined as any disease relapse within 3 months of RC [7]. The Mayo Clinic Cystectomy Registry excluded 72 of the 2,438 patients because of development of metastatic disease within 30 days of surgery [8]. Hussein et al. [9] sought to investigate the prevalence and variables associated with EOF following robot-assisted
RC EOF developed in 5\% of their patients. The lack of similar open reports of EOF in the open RC literature limits any comparison with RARC.

In the largest study on the STSM status, it was a powerful predictor of outcomes after RC. The recurrence-free survival probabilities of patients with positive versus negative STSM were 21 vs. $63 \%$ at 5 years after RC [10]. Our Group 1 patients were never free of disease and all had positive STSM. As opposed to Group 1, all Group 2 patients were free of disease after RC, albeit 7 had occult lymph node mts. Only 1 patient developed an LR and the distant failure rate was 9 out of 12 patients.

In an autopsy study, the pattern of mts in advanced BC has been analysed by Wallmeroth et al. [11]. The frequency of mts increased with local tumour extension and was higher in patients treated by $\mathrm{RC}$ than in patients without RC. For pT4 patients it was 89 vs. $79 \%$ respectively. The most frequent sites of $\mathrm{mts}$ were regional lymph nodes, liver, lung, bone and peritoneum in 90, 47, 45, 32 and $19 \%$ respectively. This corresponds to the pattern of our Group 2.

Clinical staging is the main determinant governing decision making. We found, that the level of agreement between clinical and pathological staging for EOM patients was only $10 \%$ for the primary tumour alone (T-stage) and $40 \%$ when lymph node involvement was considered upstaging (N-stage). Our rates of stage discrepancy are below the error range of clinical staging reported in the literature, which are between 27 and 62\% [12, 13]. Reasons for that are, that in some of our patients in Group 2 the location of the mts escaped conventional staging examinations (f.i. the 4 patients with brain $\mathrm{mts}$ ) and was only symptom driven. The key driver for EOM in Group 1 was the high upstaging rate to $\mathrm{pT} 4 \mathrm{~b}$ resulting from a frozen pelvis. Our experience with 11 out of 24 patients being upstaged pT4b underscores that conventional imaging techniques scan have a limited role in staging $\mathrm{BC}$ patients, In a recent meta-analysis, Gandhi et al. concluded that MRI for $<$ T4b versus pT4b sensitivity exceeded the estimated accuracy for clinical staging and should be considered potentially superior to the current standard for clinical staging $[14,15]$.

Strengths of our study are the homogeneity of this large single-centre series and the completeness of staging and follow-up. All patients were treated by high-volume surgeons under standardized conditions in terms of indications for surgery, operative characteristics, postoperative care, analysis of complications and comorbidity. There are several limitations to our study, especially the limitations that are inherent to retrospective analyses. 
The study period spans almost 25 years and the data in the present study may not represent current practice patterns. However, 769 of the patients were treated in the 1990s and 718 in the year 2000 or thereafter, which may limit the relevance of such issues.

What could have been done better for these 24 patients? What could be done better for similar patients in 2019 ?

The majority of our patients were treated before the NAC era. Furthermore our institutional treatment protocol for BC till 2009 was "surgery only," and our 10 year OS, disease-specific survival and cancer-specific survival rates of $44.3,66.8$ and $65.5 \%$ [4] compare favourably to any NAC series [16]. However, in 2019, potential improvements for a treatment of this type of disease are on the horizon. At the 2019 AUA there was a report on neoadjuvant pembrolizumab and Cis platin chemo therapy for MIBC. The pathologic non-muscle invasive rate $(\leq \mathrm{pT} 1, \mathrm{NO}, \mathrm{MO})$ was $61 \%$, the pT0 rate was $44.4 \%$. These rates warrant further study [17].

Our high upstaging proportion of patients with clinically localized disease to advanced or metastatic disease must be accepted.

Positron emission tomography combined with CT is increasingly used in clinical practice and its exact role continues to be evaluated.

All of our Group 1 patients had unanticipated findings of bulky/metastatic disease at the time of a planned curative RC. The clinical outcome and optimum management of these patients is unclear [18, 19]. Guzzo et al. [20] evaluated the clinical outcome of patients who had an aborted attempt at curative RC for intraoperative findings of metastatic disease. Sixty per cent of their patients died within the study period, $31 \%$ are alive with progression/ persistent disease, and 9\% are NED. The authors conclude when metastatic disease is discovered at RC, completing the RC should be considered [20]. Until clinical staging improves and is supported by genomic data we believe that an attempt of RC as primary treatment should be offered to Group 1 patients.

\section{Conclusions}

EOM is not negligible (1.6\%). High-risk tumours including non-urothelial BC, advanced pathological tumour stage, positive STSM, unresectable tumours, tumours with atypical mts (brain) that escape the conventional staging examinations, and a dramatic upstaging rate, but not surgical technical related factors (entry into the bladder, urine leakage from urethra, or ureter) have the leading role in EOM after open RC.

\section{Statement of Ethics}

All human subjects provided written informed consent with guarantees of confidentiality. Institutional review board approved protocol number is URO-CX-1/2009.

\section{Disclosure Statement}

The authors declare that they have no conflict of interest to disclose.

\section{References}

1 Isbarn H, Jeldres C, Zini L, Perrotte P, Baillargeon-Gagne S, Capitanio U, et al. A population based assessment of perioperative mortality after cystectomy for bladder cancer. J Urol. 2009 Jul;182(1):70-7.

2 Quek ML, Stein JP, Daneshmand S, Miranda G Thangathurai D, Roffey P, et al. A critical analysis of perioperative mortality from radical cystectomy. J Urol. 2006 Mar;175(3 Pt 1):886-9.

3 Groeben C, Koch R, Baunacke M, Borkowetz A, Wirth MP, Huber J. In-Hospital Outcomes after Radical Cystectomy for Bladder Cancer: Comparing National Trends in the United States and Germany from 2006 to 2014. Urol Int. 2019;102(3):284-92.

4 Hautmann RE, de Petriconi RC, Pfeiffer C, Volkmer BG. Radical cystectomy for urothelial carcinoma of the bladder without neoadjuvant or adjuvant therapy: long-term results in 1100 patients. Eur Urol. 2012 May;61(5):1039-47.

5 Donat SM, Siegrist T, Cronin A, Savage C, Milowsky MI, Herr HW. Radical cystectomy in octogenarians-does morbidity outweigh the potential survival benefits? J Urol. 2010 Jun;183(6):2171-7.

6 Madersbacher S, Hochreiter W, Burkhard F, Thalmann GN, Danuser H, Markwalder R, etal. Radical cystectomy for bladder cancer today-a homogeneous series without neoadjuvant therapy. J Clin Oncol. 2003 Feb;21(4):690-6.

7 Mari A, Campi R, Tellini R, Gandaglia G, Albisinni S, Abufaraj M, et al. Patterns and predictors of recurrence after open radical cystectomy for bladder cancer: a comprehensive review of the literature. World J Urol. 2018 Feb;36(2):157-70.
8 Merrill SB, Boorjian SA, Thompson $\mathrm{RH}$ Psutka SP, Cheville JC, Thapa P, et al. Oncologic surveillance following radical cystectomy: an individualized risk-based approach. World J Urol. 2017 Dec;35(12):1863-9.

9 Hussein AA, Saar M, May PR, Wijburg CJ, Richstone L, Wagner A, et al.; Collaborators. Early Oncologic Failure after RobotAssisted Radical Cystectomy: Results from the International Robotic Cystectomy Consortium. J Urol. 2017 Jun;197(6):142736.

10 Novara G, Svatek RS, Karakiewicz PI, Skinner E, Ficarra V, Fradet Y, et al. Soft tissue surgical margin status is a powerful predictor of outcomes after radical cystectomy: a multicenter study of more than 4,400 patients. J Urol. 2010 Jun;183(6):2165-70. 
11 Wallmeroth A, Wagner U, Moch H, Gasser TC, Sauter G, Mihatsch MJ. Patterns of metastasis in muscle-invasive bladder cancer (pT2-4): an autopsy study on 367 patients. Urol Int. 1999;62(2):69-75.

12 Shariat SF, Palapattu GS, Karakiewicz PI, Rogers CG, Vazina A, Bastian PJ, et al. Discrepancy between clinical and pathologic stage: impact on prognosis after radical cystectomy. Eur Urol. 2007 Jan;51(1):137-49.

13 Dutta SC, Smith JA Jr, Shappell SB, Coffey CS, Chang SS, Cookson MS. Clinical under staging of high risk nonmuscle invasive urothelial carcinoma treated with radical cystectomy. J Urol. 2001 Aug;166(2): 490-3.

14 Li R, Metcalfe M, Kukreja J, Navai N. Role of Radical Cystectomy in Non-Organ Confined
Bladder Cancer: A Systematic Review. Bladder Cancer. 2018 Jan;4(1):31-40.

15 Gandhi N, Krishna S, Booth CM, Breau RH, Flood TA, Morgan SC, et al. Diagnostic accuracy of magnetic resonance imaging for tumour staging of bladder cancer: systematic review and meta-analysis. BJU Int. 2018 Nov; 122(5):744-53.

16 Faiena I, Salmasi A, Mendhiratta N, Lenis AT, Pooli A, Drakaki A, et al. Overall survival in patients with residual disease after radical cystectomy and neoadjuvant chemotherapy. World J Urol. 2018 Nov;36(11):1825-33.

17 Kaimakliotis H, Albany C, Hoffman-Censits J, Trabulsi E, Kelly WK, Picus J, et al. A multicenter phase $1 \mathrm{~B} / 2$ study of neoadjuvant pembrolizumab and cisplatin chemotherapy for muscle invasive urothelial cancer. J Urol
Vol. 2019;201(No. 4S, Supplement, PD5203):e924.

18 Abufaraj M, Dalbagni G, Daneshmand S, Horenblas S, Kamat AM, Kanzaki R, et al. The Role of Surgery in Metastatic Bladder Cancer: A Systematic Review. Eur Urol. 2018 Apr; 73(4):543-57.

19 Tilki D, Svatek RS, Karakiewicz PI, Isbarn H, Reich O, Kassouf W, et al. Characteristics and outcomes of patients with pT4 urothelial carcinoma at radical cystectomy: a retrospective international study of 583 patients. J Urol. 2010 Jan;183(1):87-93.

20 Guzzo TJ, Rogers CG, Deng CY, Bivalacqua TJ, Palapattu GS, Bastian PJ, et al. Outcomes of patients after aborted radical cystectomy for intraoperative findings of metastatic disease. BJU Int. 2008 Dec;102(11):1539-43. 